\title{
Quantized Sampled-Data Control for T-S Fuzzy System Using Discontinuous LKF Approach
}

\author{
Shenquan Wang, Shuaiqi Chen, Wenchengyu Ji and Keping Liu* \\ College of Electrical and Electronic Engineering, Changchun University of Technology, Changchun, China
}

In this study, the stability for a class of sampled-data Takagi-Sugeno (T-S) fuzzy systems with state quantization was investigated. Using the discontinuous LyapunovKrasoskii functional (LKF) approach and the free-matrix-based integral inequality bounds processing technique, a stability condition with less conservativeness has been obtained, and the controller of the sampled-data T-S fuzzy system with the quantized state has been designed. Furthermore, based on the results, the sampled-data T-S fuzzy system without the state quantization was also discussed, and the required controller constructed. The results of two simulation examples show that both the maximum

OPEN ACCESS

Edited by: Yanzheng Zhu,

Western Sydney University, Australia

Reviewed by:

Bo Zhao,

Beijing Normal University, China

Tiao-Yang Cai,

Hebei Normal University, China

Junyi Wang,

Loughborough University,

United Kingdom

*Correspondence:

Keping Liu

liukeping@ccut.edu.cn

Specialty section:

This article was submitted to Autonomic Neuroscience,

a section of the journal

Frontiers in Neuroscience

Received: 20 January 2019

Accepted: 01 April 2019

Published: 24 April 2019

Citation:

Wang S, Chen S, Ji W and Liu K (2019) Quantized Sampled-Data Control for T-S Fuzzy System Using

Discontinuous LKF Approach. Front. Neurosci. 13:372.

doi: 10.3389/fnins.2019.00372 sampling intervals, with and without the quantized state for T-S fuzzy systems, are actually superior to the existing results.

Keywords: stabilization, T-S fuzzy systems, quantization, sampled-data system, discontinuous LKF approach

\section{INTRODUCTION}

In the real world, most physical systems and processes can be modeled mathematically as complex nonlinear systems. Because some parts of nonlinear systems are always coupled and influence each other, it is difficult to analyze and synthesize these systems. Therefore, establishing an effective and suitable control model to address this issue in nonlinear systems, is significant. In recent years, T-S fuzzy systems has been an effective method to analyze and synthesize nonlinear systems, because of the fact that the T-S fuzzy model is able to transform a complex nonlinear system into several simple linear systems with membership functions, approximating the nonlinear function smoothly with an arbitrary precision in the closed set space, which is ubiquitous in chemical processes, robotics systems, and automatic systems (Tanaka and Wang, 2004). Thus, many results on T-S fuzzy systems, analyzing nonlinear systems, have been reported in terms of various methods, including stability analysis (Lam et al., 2007; Zhao et al., 2009; Zhu et al., 2012, 2018a), controller design (Xia et al., 2010; Wu et al., 2014a,b, 2015; Liu et al., 2016; Wang et al., 2018a; Zhong et al., 2018; Zhao et al., 2019), and fault detection and filter design (Li et al., 2015; Wang et al., 2016, 2017, 2018b; Zhu et al., 2018b), etc.

At the front line of other research, there has been increasing interest in the sampled-data control system, a rapid development of digital and communication technology (Chen and Francis, 2012). The sampled-data control systems involve both continuous-time and discrete-time signals, which make the analysis and synthesis more complicated and challenging. It should be noted that the sampling period is an important issue when analyzing system stability. A larger sampling period can reduce the occupation of the communication channel, and the actuation of the controller and signal transmission. Thus, it is very important to guarantee the stability of the sampled-data system, with a sampling period as large as possible. Over the past several years, sampled-data 
control systems have drawn much attention (see Hu et al., 2007; Zhu et al., 2012; Shao et al., 2014; Wu et al., 2014a,b, 2015; Liu et al., 2016; Lee and Park, 2017; Wang et al., 2018a and the references therein), and one popular and widely used approach to analyze and synthesize sampled-data systems is the input delay approach, which is based on the representation of the sampleddata system as a continuous-time system with a delayed control input, and does not require the sampling interval to be constant (Fridman et al., 2004; Fridman, 2010; Yang et al., 2014; Li et al., 2015). The authors in $\mathrm{Hu}$ et al. (2007) designed a sampled-data controller for networked linear control, and Shao et al. (2014) researched the problem of sampling-interval-dependent stability for sampled-data systems with state quantization. Nevertheless, the results of $\mathrm{Hu}$ et al. (2007) and Shao et al. (2014) required the research system to be linear, which is impractical for many applications. Very recently, the problem of stability and stabilization for sampled-data nonlinear fuzzy systems with state quantization was investigated in Liu et al. (2016). Furthermore, the authors in Wang et al. (2018a), designed a dissipativity-based reliable controller for the sampled-data T-S fuzzy system, using the limited Bessel-Legendre inequality proposed in Liu et al. (2017), and a less conservative sufficient stabilization criterion was obtained to guarantee that the sampled-data systems are asymptotically stable. Although there are few results to study the stability of sampled-data T-S fuzzy systems, it is still necessary to decrease the conservativeness of the stability criteria further.

It is well-known that due to the limited capacity and energy consumption in the network system, it is especially important to quantize the sampled-data before transmission. However, most existing studies assume that the data transmission can be performed with infinite precision, and the impact of quantization is always ignored in the network environment. On the other hand, sampling quantization before signal transmission may lead to a limited cycle and chaos. Thus, the study of sampled-data systems with state quantization has attracted significant research attention (Fu and Xie, 2005; Niu et al., 2009; Shao et al., 2014; Liu et al., 2016; Dong et al., 2017a,b). The author in Dong et al. (2017b) studied the reliable control problem for fuzzy systems with state quantization and switched actuator failures. The output feedback control problem of the network control systems with signal quantization and packet loss has been studied in Niu et al. (2009). The stability for sampled-data systems and linear systems with state quantization, have been studied in Dong et al. (2017a), Shao et al. (2014), and Fu and Xie (2005), respectively. Up to now, to the best of authors' knowledge, little attention is given to the issue of quantized sampled-data control for T-S fuzzy systems. Thus, the aim of this work, with a focus on the stability and stabilization problem for sampleddata T-S fuzzy systems with state quantization, is to decrease the conservativeness of the stability criteria even further.

Motivated by that, this work mainly discusses the stability and stabilization control problem for nonlinear T-S fuzzy sampled-data systems with quantized states. By using the discontinuous LKF approach and free-matrix-based integral inequality boundary processing technique, stability conditions with less conservativeness are obtained for T-S fuzzy systems, with sampled-data and quantized states, and the controllers are designed accordingly. Furthermore, the stability of T-S fuzzy sampled-data systems without quantized states is also analyzed utilizing the above theoretical results, and the required sampling data controllers are designed simultaneously. The main contributions of this paper can be summarized as follows: (1) In constructing LKF aspects, it is not necessary that the discontinuous LKF approach applied in this work are positive for all time $t$, only at the sampling times $t_{k}$ and $t_{k+1}$, which can broaden the restriction in LKF. (2) In estimating the derivation of LKF, the free-matrix-based integral inequality boundary processing technique is used to provide more freedom in deriving stability for sampled-date T-S fuzzy systems. From two points of view, the conservativeness of stability conditions can be decreased for sampled-data T-S fuzzy systems with and without quantized states effectively through the methods of our design in this work.

Notations. Throughout this paper, $I$ denotes the identity matrix with appropriate dimensions. $\operatorname{Sym}(A)$ denotes $A+A^{T}$. $\mathcal{R}^{n}$ denotes the $n$ dimensional Euclidean space, and $\mathcal{R}^{n \times m}$ is the set of all $n \times m$ real matrices. $S_{+}^{n}$ denotes the $n$ dimensional symmetric positive matrices. $*$ denotes the elements below the main diagonal of a symmetric block matrix.

\section{PROBLEM FORMULATION}

A class of continuous-time nonlinear systems can be described using the following T-S fuzzy model then

The rules of plant $i$ : If $\theta_{1}(t)$ is $M_{i 1}(t)$ and $\ldots$ and $\theta_{n}(t)$ is $M_{i n}(t)$,

$$
\dot{x}(t)=A_{i} x(t)+B_{i} u(t)
$$

where $x(t) \in \mathcal{R}^{n}$ and $u(t) \in \mathcal{R}^{m}$ are the state and control input vectors of the system, respectively. $A_{i}$ and $B_{i}$ are any matrices with appropriate dimensions. $\theta(t)=\left[\theta_{1}(t) \cdots \theta_{n}(t)\right]$ is the premise variable; $M_{i j}(t)$ is the fuzzy set, with $i=1,2, \cdots, r$ as the amount of fuzzy rules. Then the above continuous time T-S fuzzy system (Equation 1) can be expressed in the following form

$$
\dot{x}(t)=\sum_{i=1}^{r} h_{i}(\theta(t))\left[A_{i} x(t)+B_{i} u(t)\right]
$$

where $h_{i}(\theta(t))$ represents the membership function and satisfies $h_{i}(\theta(t))=\omega_{i}(\theta(t)) / \sum_{i=1}^{r} \omega_{i}(\theta(t)) ; \omega_{i}(\theta(t))=\prod_{j=1}^{n} M_{i j}\left(\theta_{j}(t)\right)$, where $M_{i j}\left(\theta_{j}(t)\right)$ is the degree of membership and $\theta_{j}(t)$ belongs to the fuzzy set of $M_{i j}$, which has the following properties. For $\theta(t), \omega_{i}(\theta(t)) \geq 0$ and $\sum_{i=1}^{r} \omega_{i}(\theta(t))>0$, we can determine $h_{i}(\theta(t)) \geq 0(\forall i=1,2, \cdots, r)$ and $\sum_{i=1}^{r} h_{i}(\theta(t))=1$.

Suppose the control signal is a sequence of holding times generated by a zero-order-holder function.

$$
0=t_{0}<t_{1}<\cdots<\lim _{k \rightarrow \infty} t_{k}=+\infty
$$

This paper designs the controller based on the state feedback for T-S fuzzy systems described in (1) and uses the idea of parallel distributed compensation to share the same fuzzy set with the fuzzy model in the premise part of the designed fuzzy controller. 

then

Controller rules $j$ : If $\theta_{1}\left(t_{k}\right)$ is $M_{j 1}(t)$ and $\ldots$ and $\theta_{n}\left(t_{k}\right)$ is $M_{j n}(t)$,

$$
u(t)=K_{j} x\left(t_{k}\right), t_{k} \leq t \leq t_{k+1}, j=1,2, \cdots, r
$$

where $K_{j}$ is the state feedback gain matrix with appropriate dimension, and $x\left(t_{k}\right)$ is the discrete measurement value at the sampling time of $t_{k}$.

The logarithm quantizer is described as

$$
q(\bullet)=\left[q_{1}(\bullet) q_{2}(\bullet) \cdots q_{n}(\bullet)\right]^{T}
$$

The $m$ th sub-quantizer $q_{m}(\bullet)$ is symmetric, and therefore we have

$$
q_{m}\left(x_{m}\left(t_{k}\right)\right)=-q_{m}\left(-x_{m}\left(t_{k}\right)\right)
$$

The quantizer satisfies the following quantization criteria

$$
\begin{gathered}
\left\{ \pm \sigma_{m}^{r} \mid \sigma_{m}^{r}=\left(\rho_{m}\right)^{r} \sigma_{m}^{(0)}, m=0, \pm 1, \pm 2, \cdots\right\} \bigcup\{0\} \\
0 \leq \rho_{m}<1, \sigma_{m}^{(0)}>0
\end{gathered}
$$

where $\rho_{m}$ and $\sigma_{m}^{(0)}$ are quantizer density and initial quantization values, respectively. $q_{m}(\bullet)$ is strictly defined as follows

$$
q_{m}\left(x\left(t_{k}\right)\right)= \begin{cases}\sigma_{m}^{(r)}, \frac{\sigma_{m}^{(r)}}{1+l_{m}} \leq x_{m}\left(t_{k}\right) \leq \frac{\sigma_{m}^{(r)}}{1-l_{m}}, & \text { if } x_{m}\left(t_{k}\right)>0 \\ 0, & \text { if } x_{m}\left(t_{k}\right)=0 \\ -q_{m}\left(-x_{m}\left(t_{k}\right)\right), & \text { if } x_{m}\left(t_{k}\right)<0\end{cases}
$$

where $l_{m}=1-\rho_{m} / 1+\rho_{m}(m=1,2, \cdots, n)$ is the parameter of the quantizer. When $x_{m}\left(t_{k}\right)>0$, the following relationship is established

$$
\left(1-l_{m}\right) x_{m}\left(t_{k}\right) \leq \sigma_{m}^{(r)} \leq\left(1+l_{m}\right) x_{m}\left(t_{k}\right)
$$

For $x_{m}\left(t_{k}\right)<0$, the following is satisfied

$$
\left(1+l_{m}\right) x_{m}\left(t_{k}\right) \leq \sigma_{m}^{(r)} \leq\left(1-l_{m}\right) x_{m}\left(t_{k}\right)
$$

Therefore, the quantizer can be expressed as

$$
q\left(x\left(t_{k}\right)\right)=x\left(t_{k}\right)+f\left(x\left(t_{k}\right)\right)
$$

where

$$
\begin{aligned}
& f\left(x\left(t_{k}\right)\right)=\left[f_{1}\left(x\left(t_{k}\right)\right) f_{2}\left(x\left(t_{k}\right)\right) \cdots f_{n}\left(x\left(t_{k}\right)\right)\right]^{T} \\
& -l_{m}\left[x_{m}\left(t_{k}\right)\right]^{2} \leq x_{m}\left(t_{k}\right) f_{m}\left(x\left(t_{k}\right)\right) \leq l_{m}\left[x_{m}\left(t_{k}\right)\right]^{2}
\end{aligned}
$$

Hence, the overall state feedback controller with the quantized state can be designed

$$
u(t)=\sum_{j=1}^{r} h_{j}\left(\theta\left(t_{k}\right)\right) K_{j}\left[x\left(t_{k}\right)+f\left(x\left(t_{k}\right)\right)\right]
$$

Suppose the distance between two consecutive sampling instants belongs to an interval, and then for all $k>0$ there exists

$$
t_{k+1}-t_{k}=h_{k}, h_{k} \in\left[h_{L}, h_{U}\right]
$$

where $h_{L}$ and $h_{U}$ are known constants satisfying $0 \leq h_{L} \leq h_{U}$. Combining Equations (1)-(6), the T-S fuzzy sampled-data system with state quantization can be obtained as follows

$$
\dot{x}(t)=\sum_{i=1}^{r} \sum_{j=1}^{r} h_{i}(\theta(t)) h_{j}\left(\theta\left(t_{k}\right)\right)\left[A_{i} x(t)+B_{i} K_{j}\left(x\left(t_{k}\right)+f\left(x\left(t_{k}\right)\right)\right)\right]
$$

The following lemma is important for further analysis.

Lemma 1. Lee and Park (2017) (Free-Matrix-Based Integral Inequality) For given matrices $R \in S_{+}^{n}, N_{1}, N_{2} \in \mathcal{R}^{3 n \times n}$, the following inequality holds for any continuously differentiable function $x(t)$ in $[a, b] \in \mathcal{R}^{n}$

$$
-\int_{\alpha}^{\beta} \dot{x}^{T}(s) R \dot{x}(s) d s \leq \phi^{T}(\alpha, \beta) \Psi(\alpha, \beta) \phi(\alpha, \beta)
$$

where

$$
\begin{aligned}
& \Psi(\alpha, \beta)=(\beta-\alpha)\left(N_{1} R^{-1} N_{1}^{T}+\frac{(\beta-\alpha)^{2}}{3} N_{2} R^{-1} N_{2}^{T}+Z_{1}\right)+Z_{2} \\
& \phi(\alpha, \beta)=\left[\begin{array}{lll}
x^{T}(\beta) & x^{T}(\alpha) \int_{\alpha}^{\beta} x^{T}(s) d s
\end{array}\right]^{T} \\
& Z_{1}=-\operatorname{Sym}\left[\begin{array}{lll}
N_{2} & N_{2} & 0
\end{array}\right] \\
& Z_{2}=\operatorname{Sym}\left[\begin{array}{lll}
N_{1} & -N_{1} & 2 N_{2}
\end{array}\right]
\end{aligned}
$$

Remark 1. Note that the free-matrix-based integral inequality in Lemma 1 provides more freedom in deriving stability for sampled-date T-S fuzzy systems. So, it provides the possibility of finding a tight bound for $-\int_{\alpha}^{\beta} \dot{x}^{T}(s) R \dot{x}(s) d s$. On the other hand, the augmented vector $\phi(\alpha, \beta)$ includes $\int_{\alpha}^{\beta} x^{T}(s) d s$ instead of $\frac{1}{\beta-\alpha} \int_{\alpha}^{\beta} x^{T}(s) d s$, and this avoids appearance of the term $(t-$ $\left.t_{k}\right) \phi\left(t_{k}, t\right)$ in sampled-data systems, which is actually much easier to handle.

\section{MAIN RESULT}

\subsection{Stability Analysis of the Sampled-Data T-S Fuzzy System With State Quantization}

In this section, the asymptotic stability conditions of T-S fuzzy systems with state quantization are analyzed.

For the convenience of system analysis and design, we define

$$
\begin{aligned}
e_{i} & =\left[\begin{array}{lll}
0_{n \times(i-1) n} & I_{n} & 0_{n \times(5-i) n}
\end{array}\right], \\
\xi^{T}(t) & =\left[\begin{array}{llll}
x^{T}(t) & x^{T}\left(t_{k}\right) & \int_{t_{k}}^{t} x^{T}(s) d s & \dot{x}^{T}(t) f^{T}\left(x\left(t_{k}\right)\right)
\end{array}\right]
\end{aligned}
$$


where $e_{i}$ is defined as the block entry matrix. Other notations are defined as

$$
\begin{aligned}
& \eta_{1}^{T}(t)=\left[x^{T}(t)-x^{T}\left(t_{k}\right) \int_{t_{k}}^{t} x^{T}(s) d s f^{T}\left(x\left(t_{k}\right)\right)\right] \\
& \eta_{2}^{T}(t)=\left[x^{T}\left(t_{k}\right) \dot{x}^{T}(t) f^{T}\left(x\left(t_{k}\right)\right)\right] \\
& \eta_{3}^{T}(t)=\left[\begin{array}{lll}
\dot{x}^{T}(t) & x^{T}(t) & 0
\end{array}\right] \\
& \eta_{4}^{T}(t)=\left[\begin{array}{lll}
x^{T}(t) & \left.x^{T}\left(t_{k}\right) \int_{t_{k}}^{t} x^{T}(s) d s\right]
\end{array}\right. \\
& \eta_{5}^{T}(t)=\left[\begin{array}{lll}
\dot{x}^{T}(t) & 0 & x^{T}(t)
\end{array}\right] \\
& \Pi_{1}^{T}=\left[\begin{array}{lll}
e_{1}^{T}-e_{2}^{T} & e_{3}^{T} & e_{5}^{T}
\end{array}\right] \\
& \Pi_{2}^{T}=\left[\begin{array}{lll}
e_{2}^{T} & e_{4}^{T} & e_{5}^{T}
\end{array}\right] \\
& \Pi_{3}^{T}=\left[\begin{array}{lll}
e_{4}^{T} & e_{1}^{T} & 0
\end{array}\right] \\
& \Pi_{4}^{T}=\left[\begin{array}{lll}
e_{2}^{T} & e_{2}^{T} & e_{3}^{T}
\end{array}\right] \\
& \Pi_{5}^{T}=\left[\begin{array}{lll}
e_{4}^{T} & 0 & e_{1}^{T}
\end{array}\right]
\end{aligned}
$$

Theorem 1. For given scalars $h_{L}$ and $h_{U}$, satisfying $0 \leq h_{L} \leq h_{U}$, system (Equation 7) is asymptotically stable, if there are some symmetric positive definite matrices $P \in S_{+}^{n}, X_{1}, Q \in S_{+}^{3 n}$, $R \in S_{+}^{7 n}$; a diagonal matrix $D \in S_{+}^{n}$; any matrices $G_{1}, G_{2}, X_{2}$, $N_{1}, N_{2}$ with appropriate dimensions, and for any $i \geq 1$ and $j \leq r$, the following linear matrix inequalities (LMIs) hold

$$
\begin{gathered}
\Phi_{1}+h_{k} \Phi_{2}<0 \\
{\left[\begin{array}{ccc}
\Phi_{1}+h_{k} \Phi_{3} & \Phi_{12} & \Phi_{13} \\
* & -Q_{4} & 0 \\
* & * & -3 Q_{4}
\end{array}\right]<0}
\end{gathered}
$$

where

$$
\begin{gathered}
\Phi_{1}=\operatorname{Sym}\left(e_{1}^{T} P e_{4}\right)-\Pi_{1}^{T} X_{1} \Pi_{1}-\operatorname{Sym}\left(e_{3}^{T} X_{2} e_{2}\right) \\
-\operatorname{Sym}\left(\left[\begin{array}{l}
e_{2} \\
e_{5}
\end{array}\right]^{T}\left[\begin{array}{l}
Q_{2} \\
Q_{5}
\end{array}\right]\left(e_{1}-e_{2}\right)\right)+\Pi_{4}^{T} \Re_{1} \Pi_{4} \\
+\operatorname{Sym}\left(\Pi_{4}^{T} \Re_{2} \Pi_{5}\right)+e_{4}^{T} R_{6} e_{4}+\Pi_{4}^{T} Z_{2} \Pi_{4} \\
+\operatorname{Sym}\left(\left(e_{1}^{T} G_{1}+e_{4}^{T} G_{2}\right) \Psi\right) \\
-\operatorname{Sym}\left[\left(e_{5}+L e_{2}\right)^{T} D\left(e_{5}-L e_{2}\right)\right] \\
\Phi_{3}=-\left[\begin{array}{c}
e_{2} \\
e_{5}
\end{array}\right]^{T}\left[\begin{array}{cc}
Q_{1} & Q_{3} \\
* & Q_{6}
\end{array}\right]\left[\begin{array}{l}
e_{2} \\
e_{5}
\end{array}\right]+\operatorname{Sym}\left(\Pi_{4}^{T} \Re_{1} \Pi_{5}\right)+\Pi_{4}^{T} Z_{1} \Pi_{4} \\
\Phi_{12}=\sqrt{h_{k}} \Pi_{4}^{T} N_{1} \\
\Phi_{13}=h_{U} \sqrt{h_{k}} \Pi_{4}^{T} N_{2}
\end{gathered}
$$

with

$$
\Re_{1}=R_{1}+\frac{h_{U}^{2}}{3} R_{4}-\operatorname{Sym}\left[\begin{array}{lll}
R_{5} & R_{5} & 0
\end{array}\right]
$$

$$
\begin{aligned}
& \Re_{2}=\operatorname{Sym}\left[\begin{array}{lll}
R_{3} & -R_{3} & 2 R_{5}
\end{array}\right] \\
& \Psi=\left[\begin{array}{lllll}
A_{i} & B_{i} K_{j} & 0 & -I & B_{i} K_{j}
\end{array}\right] \\
& Q=\left[\begin{array}{ccc}
Q_{1} & Q_{2} & Q_{3} \\
* & Q_{4} & Q_{5} \\
* & * & Q_{6}
\end{array}\right] \\
& R=\left[\begin{array}{ccc}
R_{1} & R_{2} & R_{3} \\
* & R_{4} & R_{5} \\
* & * & R_{6}
\end{array}\right]
\end{aligned}
$$

and $Z_{1}, Z_{2}$ have been defined in Lemma 1 .

Proof: The novel discontinuous LKF in this work is constructed as follows

$$
V(t)=V_{1}(t)+V_{2}(t)+V_{3}(t) \quad t \in\left[t_{k}, t_{k+1}\right]
$$

where

$$
\begin{aligned}
V_{1}(t) & =x^{T}(t) P x(t) \\
V_{2}(t) & =\left(t_{k+1}-t\right)\left(\eta_{1}^{T}(t) X_{1} \eta_{1}(t)+\operatorname{Sym}\left(\int_{t_{k}}^{t} x^{T}(s) d s X_{2} x\left(t_{k}\right)\right)\right. \\
& \left.+\int_{t_{k}}^{t} \eta_{2}^{T}(s) Q \eta_{2}(s) d s\right) \\
V_{3}(t) & =\eta_{4}^{T}(t) \Re \eta_{4}(t)+\int_{t_{k}}^{t} \dot{x}^{T}(s) R_{6} \dot{x}(s) d s
\end{aligned}
$$

with

$$
\Re=\left(t-t_{k}\right) \Re_{1}+\Re_{2}
$$

Remark 2. It should be noted that the constructed LKF (Equation 10) of this work is the discontinuous Lyapunov functional, as $V_{3}(t)$ is actually a time-dependent discontinuous term. Moreover, it is not necessary that $V_{1}(t)$ and $V_{2}(t)$ in LKF (Equation 10) are positive for all time $t$, but only positive at the sampling times $t_{k}$ and $t_{k+1}$. All this can decrease the conservativeness of stability conditions effectively.

The derivative of $V_{1}(t), V_{2}(t)$, and $V_{3}(t)$ can, respectively, be deduced as

$$
\dot{V}_{1}(t)=2 x^{T}(t) P \dot{x}(t)
$$

$$
\begin{array}{r}
\dot{V}_{2}(t)=-\eta_{1}^{T}(t) X_{1} \eta_{1}(t)-\operatorname{sym}\left(\int_{t_{k}}^{t} x^{T}(s) d s X_{2} x\left(t_{k}\right)\right) \\
-\operatorname{Sym}\left(\left[\begin{array}{c}
x\left(t_{k}\right) \\
f\left(x\left(t_{k}\right)\right)
\end{array}\right]^{T}\left[\begin{array}{c}
Q_{2} \\
Q_{5}
\end{array}\right]\left(x(t)-x\left(t_{k}\right)\right)\right) \\
-\left(t-t_{k}\right)\left[\begin{array}{c}
x\left(t_{k}\right) \\
f\left(x\left(t_{k}\right)\right)
\end{array}\right]^{T}\left[\begin{array}{cc}
Q_{1} & Q_{3} \\
* & Q_{6}
\end{array}\right]\left[\begin{array}{c}
x\left(t_{k}\right) \\
f\left(x\left(t_{k}\right)\right)
\end{array}\right] \\
-\int_{t_{k}}^{t} \dot{x}^{T}(s) Q_{4} \dot{x}(s) d s \\
+\left(t_{k+1}-t\right)\left(\operatorname{Sym}\left(\eta_{1}^{T}(t) X_{1} \eta_{3}(t)\right)\right. \\
\left.+\operatorname{Sym}\left(x^{T}(t) X_{2} x\left(t_{k}\right)\right)+\eta_{2}^{T}(t) Q \eta_{2}(t)\right)
\end{array}
$$




$$
\dot{V}_{3}(t)=\eta_{4}^{T}(t) \Re_{1} \eta_{4}(t)+\operatorname{Sym}\left(\eta_{4}^{T}(t) \Re \eta_{5}(t)\right)+\dot{x}^{T}(t) R_{6} \dot{x}(t)
$$

By Lemma 1, we obtain the inequality

$$
\begin{gathered}
-\int_{t_{k}}^{t} \dot{x}^{T}(s) Q_{4} \dot{x}(s) d s \leq \eta_{4}^{T}(t)\left(\left(t-t_{k}\right)\left(N_{1} Q_{4}^{-1} N_{1}^{T}+\frac{\left(t-t_{k}\right)^{2}}{3} N_{2} Q_{4}^{-1} N_{2}^{T}+Z_{1}\right)+Z_{2}\right) \\
\eta_{4}(t)
\end{gathered}
$$

Based on the closed-loop system (Equation 7), the following equality holds

$$
\begin{aligned}
& \operatorname{Sym}\left(x^{T}(t) G_{1}+\dot{x}^{T}(t) G_{2}\right) \times \\
& \qquad\left(-\dot{x}(t)+\sum_{i=1}^{r} \sum_{j=1}^{r} h_{i}(\theta(t)) h_{j}\left(\theta\left(t_{k}\right)\right)\left[A_{i} x(t)+B_{i} K_{j} x\left(t_{k}\right)+B_{i} K_{j} f\left(x\left(t_{k}\right)\right)\right]\right)=0,
\end{aligned}
$$

and it can be further written as

$$
\begin{array}{r}
\sum_{i=1}^{r} \sum_{j=1}^{r} h_{i}(\theta(t)) h_{j}\left(\theta\left(t_{k}\right)\right) \operatorname{Sym}\left(x^{T}(t) G_{1}+\dot{x}^{T}(t) G_{2}\right) \\
\left(\left[A_{i} B_{i} K_{j} 0-I B_{i} K_{j}\right] \xi(t)\right)=0
\end{array}
$$

From Equation (4), for the diagonal matrix $D>0$, it holds that

$$
-\operatorname{Sym}\left(\left[f\left(x\left(t_{k}\right)\right)+L x\left(t_{k}\right)\right]^{T} D\left[f\left(x\left(t_{k}\right)\right)-L x\left(t_{k}\right)\right]\right) \geq 0
$$

Then combining (Equations 11-16), an upper bound of $\dot{V}(t)$ can be obtained as follows

$$
\begin{array}{r}
\dot{V}(t) \leq \sum_{i=1}^{r} \sum_{j=1}^{r} h_{i}(\theta(t)) h_{j}\left(\theta\left(t_{k}\right)\right) \xi^{T}(t) \\
\left(\frac{t_{k+1}-t}{h_{k}}\left(\Phi_{1}+h_{k} \Phi_{2}\right)+\frac{t-t_{k}}{h_{k}}\left(\Phi_{1}+h_{k} \breve{\Phi}_{3}\right)\right) \xi(t)
\end{array}
$$

where

$$
\begin{aligned}
\breve{\Phi}_{3}= & -\left[\begin{array}{l}
e_{2} \\
e_{5}
\end{array}\right]^{T}\left[\begin{array}{ll}
Q_{1} & Q_{3} \\
* & Q_{6}
\end{array}\right]\left[\begin{array}{l}
e_{2} \\
e_{5}
\end{array}\right]+\operatorname{Sym}\left(\Pi_{4}^{T} \Re_{1} e_{2}\right)+\Pi_{4}^{T} Z_{1} \Pi_{4} \\
& +\Pi_{4}^{T}\left(N_{1} Q_{4}^{-1} N_{1}^{T}+\frac{\left(t-t_{k}\right)^{2}}{3} N_{2} Q_{4}^{-1} N_{2}^{T}\right) \Pi_{4}
\end{aligned}
$$

with the following two equalities

$$
\begin{aligned}
\Phi_{1}+h_{k} \Phi_{2} & =\frac{h_{U}-h_{k}}{h_{U}-h_{L}}\left(\Phi_{1}+h_{L} \Phi_{2}\right)+\frac{h_{k}-h_{L}}{h_{U}-h_{L}}\left(\Phi_{1}+h_{U} \Phi_{2}\right) \\
\Phi_{1}+h_{k} \breve{\Phi}_{3} & =\frac{h_{U}-h_{k}}{h_{U}-h_{L}}\left(\Phi_{1}+h_{L} \breve{\Phi}_{3}\right)+\frac{h_{k}-h_{L}}{h_{U}-h_{L}}\left(\Phi_{1}+h_{U} \breve{\Phi}_{3}\right)
\end{aligned}
$$

Therefore, using Schur Complement, Equations (8) and (9) are equivalent to $\dot{V}(t)<0$. This completes the proof.
Additionally, Theorem 1 has provided the stability results for T-S fuzzy sampled-data systems (Equation 7) with state quantization, and, the following Theorem 2 will be given in order to obtain the sampled-data controller.

Theorem 2. For given scalars $h_{L}$ and $h_{U}$, satisfying $0 \leq h_{L} \leq$ $h_{U}$, system (Equation 7) is asymptotically stable, if there are some symmetric positive definite matrices $\bar{P} \in S_{+}^{n}, \bar{X}_{1}, \bar{Q} \in S_{+}^{3 n}$, $\bar{R} \in S_{+}^{7 n}$; diagonal matrix $\bar{D} \in S_{+}^{n}$; any matrices $G, T_{j}, \bar{X}_{2}, \bar{N}_{1}$, $\bar{N}_{2}$ with appropriate dimensions, and for any $i \geq 1$ and $j \leq r$, the following LMIs hold

$$
\bar{\Phi}_{1}+h_{k} \bar{\Phi}_{2}<0
$$

$$
\left[\begin{array}{ccc}
\bar{\Phi}_{1}+h_{k} \bar{\Phi}_{3} & \bar{\Phi}_{12} & \bar{\Phi}_{13} \\
* & -\bar{Q}_{4} & 0 \\
* & * & -3 \bar{Q}_{4}
\end{array}\right]<0
$$

where

$$
\begin{gathered}
\bar{\Phi}_{1}=\operatorname{Sym}\left(e_{1}^{T} \bar{P} e_{4}\right)-\Pi_{1}^{T} \bar{X}_{1} \Pi_{1}-\operatorname{Sym}\left(e_{3}^{T} \bar{X}_{2} e_{2}\right) \\
-\operatorname{Sym}\left(\left[\begin{array}{l}
e_{2} \\
e_{5}
\end{array}\right]^{T}\left[\begin{array}{l}
\bar{Q}_{2} \\
\bar{Q}_{5}
\end{array}\right]\left(e_{1}-e_{2}\right)\right)+\Pi_{4}^{T} \bar{\Re}_{1} \Pi_{4} \\
+\operatorname{Sym}\left(\Pi_{4}^{T} \bar{\Re}_{2} \Pi_{5}\right)+e_{4}^{T} \bar{R}_{6} e_{4}+\Pi_{4}^{T} \bar{Z}_{2} \Pi_{4} \\
+\operatorname{Sym}\left(\left(e_{1}^{T}+\varepsilon e_{4}^{T}\right) \bar{\Psi}\right) \\
-\operatorname{Sym}\left[\left(e_{5}+\operatorname{Le}\right)^{T} \bar{D}\left(e_{5}-L e_{2}\right)\right] \\
\bar{\Phi}_{2}=\operatorname{Sym}\left(\Pi_{1}^{T} \bar{X}_{1} \Pi_{3}\right)+\operatorname{Sym}\left(e_{1}^{T} \bar{X}_{2} e_{2}\right)+\Pi_{2}^{T} \bar{Q} \Pi_{2} \\
\bar{\Phi}_{3}=-\left[\begin{array}{c}
e_{2} \\
e_{5}
\end{array}\right]^{T}\left[\begin{array}{c}
\bar{Q}_{1} \\
\bar{Q}_{3} \\
\bar{Q}_{6}
\end{array}\right]\left[\begin{array}{l}
e_{2} \\
e_{5}
\end{array}\right]+\operatorname{Sym}\left(\Pi_{4}^{T} \bar{\Re}_{1} \Pi_{5}\right)+\Pi_{4}^{T} \bar{Z}_{1} \Pi_{4} \\
\bar{\Phi}_{12}=\sqrt{h_{k}} \Pi_{4}^{T} \bar{N}_{1} \\
\bar{\Phi}_{13}=h_{U} \sqrt{h_{k}} \Pi_{4}^{T} \bar{N}_{2}
\end{gathered}
$$

with

$$
\begin{aligned}
& \bar{Z}_{1}=-\operatorname{Sym}\left[\begin{array}{lll}
\bar{N}_{2} & \bar{N}_{2} & 0
\end{array}\right] \\
& \bar{Z}_{2}=\operatorname{Sym}\left[\begin{array}{lll}
\bar{N}_{1} & -\bar{N}_{1} & 2 \bar{N}_{2}
\end{array}\right] \\
& \bar{\Re}=\left(t-t_{k}\right) \bar{\Re}_{1}+\bar{\Re}_{2} \\
& \bar{\Re}_{1}=\bar{R}_{1}+\frac{h_{U}^{2}}{3} \bar{R}_{4}-\operatorname{Sym}\left[\begin{array}{lll}
\bar{R}_{5} & \bar{R}_{5} & 0
\end{array}\right] \\
& \bar{\Re}_{2}=\operatorname{Sym}\left[\begin{array}{lll}
\bar{R}_{3} & -\bar{R}_{3} & 2 \bar{R}_{5}
\end{array}\right] \\
& \bar{\Psi}=\left[\begin{array}{lllll}
A_{i} G^{T} & B_{i} T_{j} & 0 & -G^{T} & B_{i} T_{j}
\end{array}\right]
\end{aligned}
$$




$$
\begin{gathered}
\bar{Q}=\left[\begin{array}{ccc}
\bar{Q}_{1} & \bar{Q}_{2} & \bar{Q}_{3} \\
* & \bar{Q}_{4} & \bar{Q}_{5} \\
* & * & \bar{Q}_{6}
\end{array}\right] \\
\bar{R}=\left[\begin{array}{ccc}
\bar{R}_{1} & \bar{R}_{2} & \bar{R}_{3} \\
* & \bar{R}_{4} & \bar{R}_{5} \\
* & * & \bar{R}_{6}
\end{array}\right]
\end{gathered}
$$

The gain matrix $K_{j}$ of the sampled-data controller with state quantization is defined as $K_{j}=T_{j} G^{-T}$.

Proof: Define

$$
\begin{aligned}
G & =G_{1}^{-1}, G_{2}=\varepsilon G_{1}, \bar{P}=G P G^{T}, \bar{X}_{2}=G X_{2} G^{T} \\
\bar{R} & =\operatorname{diag}\{G, G, G, G, G, G, G\} \operatorname{Rdiag}\{G, G, G, G, G, G, G\}^{T} \\
\bar{N}_{2} & =\operatorname{diag}\{G, G, G\} N_{2} G^{T}, \bar{N}_{1}=\operatorname{diag}\{G, G, G\} N_{1} G^{T}, \\
\bar{D} & =G D G^{T} \\
\bar{Q} & =\operatorname{diag}\{G, G, G\} Q \operatorname{diag}\{G, G, G\}^{T}, \\
\bar{X}_{1} & =\operatorname{diag}\{G, G, G\} X_{1} \operatorname{diag}\{G, G, G\}^{T}
\end{aligned}
$$

Equation (8) is pre- and post-multiplied by diag $\{G, G, G, G, G\}$ and its transpose, respectively. Equation (9) is pre- and post-multiplied by $\operatorname{diag}\{G, G, G, G, G, G, G\}$ and its transpose, respectively. We accordingly obtain Equations (18) and (19). This completes the proof.

Remark 3. Theorems 1 and 2 provide the stability condition and the controller design for the sampled-data T-S fuzzy system with the quantized state, using the discontinuous LKF approach. If we do not consider the impact of the state quantization in system (Equation 7), $f\left(x\left(t_{k}\right)\right)$ in $V_{2}(t)$ could be eliminated, and the result can be degenerated for the case without the quantized state, which will be discussed in the next section.

Remark 4. In order to obtain less conservativeness sufficient stability condition for sampled-data T-S fuzzy systems with state quantization, some other methods combined with freematrix-based integral inequality bounds processing technique could be used, such as parameter-dependent LKF and two-sided looped-functional.

\subsection{Stability Analysis of Sampled-Data T-S Fuzzy System Without State Quantization}

In this section, the impact of the quantized state is not considered, and the T-S fuzzy sampled-data system then becomes

$$
\dot{x}(t)=\sum_{i=1}^{r} \sum_{j=1}^{r} h_{i}(\theta(t)) h_{j}\left(\theta\left(t_{k}\right)\right)\left[A_{i} x(t)+B_{i} K_{j}\left(x\left(t_{k}\right)\right)\right]
$$

Define

$$
\begin{gathered}
\tilde{e}_{i}=\left[\begin{array}{lll}
0_{n \times(i-1) n} & I_{n} & 0_{n \times(4-i) n}
\end{array}\right], \\
\tilde{\xi}^{T}(t)=\left[\begin{array}{lll}
x^{T}(t) & x^{T}\left(t_{k}\right) \int_{t_{k}}^{t} x^{T}(s) d s \dot{x}^{T}(t)
\end{array}\right]^{T} \\
\tilde{\eta}_{1}^{T}(t)=\left[\begin{array}{lll}
x^{T}(t)-x^{T}\left(t_{k}\right) \int_{t_{k}}^{t} x^{T}(s) d s
\end{array}\right]
\end{gathered}
$$

$$
\begin{gathered}
\tilde{\eta}_{2}^{T}(t)=\left[\begin{array}{ll}
x^{T}\left(t_{k}\right) & \dot{x}^{T}(t)
\end{array}\right] \\
\tilde{\eta}_{3}^{T}(t)=\left[\begin{array}{ll}
\dot{x}^{T}(t) & x^{T}(t)
\end{array}\right] \\
\tilde{\eta}_{4}^{T}(t)=\left[\begin{array}{lll}
x^{T}(t) & x^{T}\left(t_{k}\right) & \int_{t_{k}}^{t} x^{T}(s) d s
\end{array}\right] \\
\tilde{\eta}_{5}^{T}(t)=\left[\begin{array}{lll}
\dot{x}^{T}(t) & 0 & x^{T}(t)
\end{array}\right] \\
\tilde{\Pi}_{1}^{T}=\left[\begin{array}{lll}
\tilde{e}_{1}^{T}-\tilde{e}_{2}^{T} & \tilde{e}_{3}^{T}
\end{array}\right] \\
\tilde{\Pi}_{2}^{T}=\left[\begin{array}{ll}
\tilde{e}_{2}^{T} & \tilde{e}_{4}^{T}
\end{array}\right] \\
\tilde{\Pi}_{3}^{T}=\left[\begin{array}{lll}
\tilde{e}_{4}^{T} & \tilde{e}_{1}^{T}
\end{array}\right] \\
\tilde{\Pi}_{4}^{T}=\left[\begin{array}{lll}
\tilde{e}_{2}^{T} & \tilde{e}_{2}^{T} & \tilde{e}_{3}^{T}
\end{array}\right] \\
\tilde{\Pi}_{5}^{T}=\left[\begin{array}{lll}
\tilde{e}_{4}^{T} & 0 & \tilde{e}_{1}^{T}
\end{array}\right]
\end{gathered}
$$

Now, using the same method used in Theorem 1, we have the following Corollary without considering the state quantization.

Corollary 1. For given scalars $h_{L}$ and $h_{U}$ with $0 \leq h_{L} \leq h_{U}$, the system (Equation 20) is asymptotically stable, if there are some symmetric positive definite matrices $P \in S_{+}^{n}, X_{1}, \tilde{Q} \in S_{+}^{2 n}$, $R \in S_{+}^{7 n}$ and any metrics $G_{1}, G_{2}, X_{2}, N_{1}, N_{2}$ with appropriate dimensions, and for any $i \geq 1$ and $j \leq r$, the following LMIs hold

$$
\Upsilon_{1}+h_{k} \Upsilon_{2}<0
$$

$$
\left[\begin{array}{ccc}
\Upsilon_{1}+h_{k} \Upsilon_{3} & \Upsilon_{12} & \Upsilon_{13} \\
* & -\tilde{Q}_{3} & 0 \\
* & * & -3 \tilde{Q}_{3}
\end{array}\right]<0
$$

where

$$
\begin{array}{r}
\Upsilon_{1}=\operatorname{Sym}\left(\tilde{e}_{1}^{T} P \tilde{e}_{4}\right)-\tilde{\Pi}_{1}^{T} X_{1} \tilde{\Pi}_{1}-\operatorname{Sym}\left(\tilde{e}_{3}^{T} X_{2} \tilde{e}_{2}\right) \\
-\operatorname{Sym}\left(\left(\tilde{e}_{2}^{T} \tilde{Q}_{2}\right)\left(\tilde{e}_{1}-\tilde{e}_{2}\right)\right)+\Pi_{4}^{T} \Re_{1} \Pi_{4} \\
+\operatorname{Sym}\left(\Pi_{4}^{T} \Re_{2} \Pi_{5}\right)+\tilde{e}_{4}^{T} R_{6} \tilde{e}_{4}+\Pi_{4}^{T} Z_{2} \Pi_{4} \\
+\operatorname{Sym}\left(\left(\tilde{e}_{1}^{T} G_{1}+\tilde{e}_{4}^{T} G_{2}\right) \tilde{\Psi}\right) \\
\Upsilon_{2}=\operatorname{Sym}\left(\tilde{\Pi}_{1}^{T} X_{1} \tilde{\Pi}_{3}\right)+\operatorname{Sym}\left(\tilde{e}_{1}^{T} X_{2} \tilde{e}_{2}\right)+\tilde{\Pi}_{2}^{T} \tilde{Q}_{2} \tilde{\Pi}_{2} \\
\Upsilon_{3}=-\tilde{e}_{2}^{T} \tilde{Q}_{1} \tilde{e}_{2}+\operatorname{Sym}\left(\Pi_{4}^{T} \Re_{1} \Pi_{5}\right)+\Pi_{4}^{T} Z_{1} \Pi_{4} \\
\Upsilon_{12}=\sqrt{h_{k}} \Pi_{4}^{T} N_{1} \\
\Upsilon_{13}=h_{U} \sqrt{h_{k}} \Pi_{4}^{T} N_{2}
\end{array}
$$

with

$$
\tilde{\Psi}=\left[\begin{array}{llll}
A_{i} & B_{i} K_{j} & 0 & -I
\end{array}\right]
$$




$$
\tilde{Q}=\left[\begin{array}{cc}
\tilde{Q}_{1} & \tilde{Q}_{2} \\
* & \tilde{Q}_{3}
\end{array}\right]
$$

where $Z_{1}, Z_{2}, R, \Re_{1}$ and $\Re_{2}$ have been defined in Theorem 1 .

Furthermore, the following Corollary regarding the sampleddata controllers design can be derived using a similar method used in Theorem 2.

Corollary 2. For given scalars $h_{L}$ and $h_{U}$ with $0 \leq h_{L} \leq$ $h_{U}$, system (Equation 20) is asymptotically stable, if there exist symmetric positive definite matrices $\bar{P} \in S_{+}^{n}, \bar{X}_{1}, \hat{Q} \in S_{+}^{2 n}, \bar{R} \in S_{+}^{7 n}$ and any matrix $G, T_{j}, \bar{X}_{2}, \bar{N}_{1}, \bar{N}_{2}$ with appropriate dimensions, and for any $1 \leq i$, and $j \leq r$, the following LMIs hold

$$
\bar{\Upsilon}_{1}+h_{k} \bar{\Upsilon}_{2}<0
$$

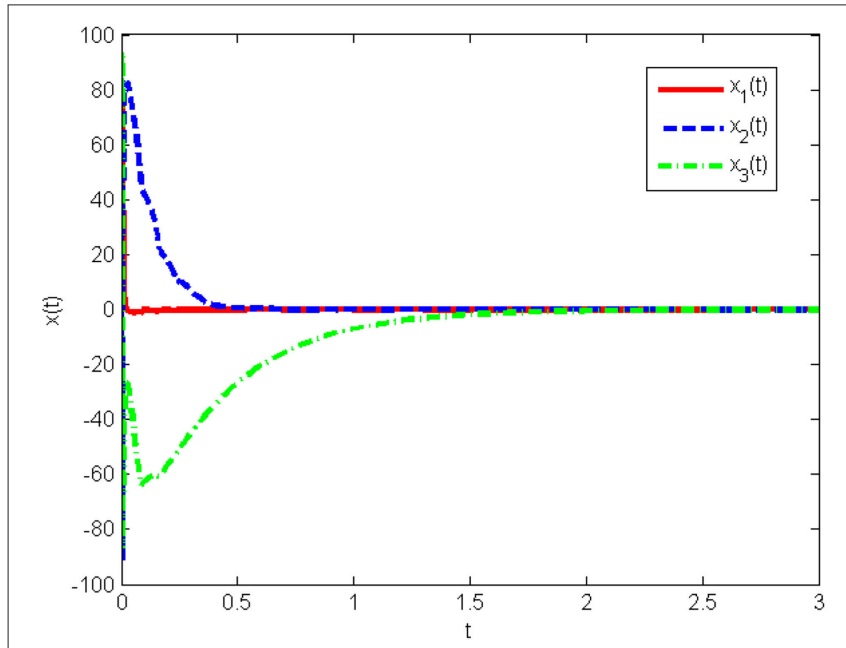

FIGURE 1 | State responses of system (Equation 25) with state quantization in Example 1.

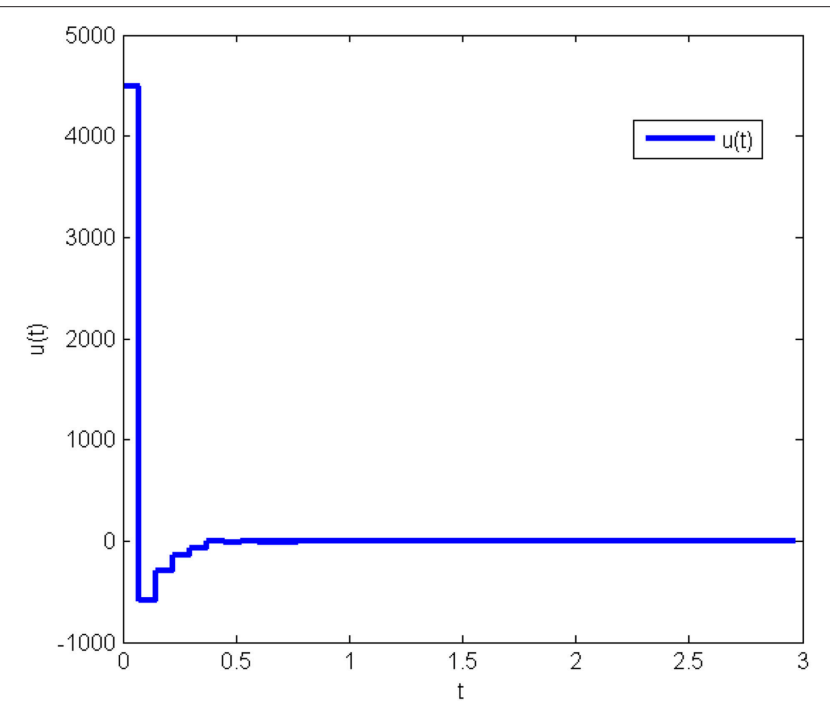

FIGURE 2 | Control input of system (Equation 25) with state quantization in Example 1.

$$
\left[\begin{array}{ccc}
\bar{\Upsilon}_{1}+h_{k} \bar{\Upsilon}_{3} & \bar{\Upsilon}_{12} & \bar{\Upsilon}_{13} \\
* & -\hat{Q}_{3} & 0 \\
* & * & -3 \hat{Q}_{3}
\end{array}\right]<0
$$

where

$$
\begin{array}{r}
\bar{\Upsilon}_{1}=\operatorname{Sym}\left(\tilde{e}_{1}^{T} \bar{P} \tilde{e}_{4}\right)-\tilde{\Pi}_{1}^{T} \bar{X}_{1} \tilde{\Pi}_{1}-\operatorname{Sym}\left(\tilde{e}_{3}^{T} \bar{X}_{2} \tilde{e}_{2}\right) \\
-\operatorname{Sym}\left(\left(\tilde{e}_{2}^{T} \hat{Q}_{2}\right)\left(\tilde{e}_{1}-\tilde{e}_{2}\right)\right)+\Pi_{4}^{T} \bar{\Re}_{1} \Pi_{4} \\
+\operatorname{Sym}\left(\Pi_{4}^{T} \bar{\Re}_{2} \Pi_{5}\right)+\tilde{e}_{4}^{T} \bar{R}_{6} \tilde{e}_{4}+\Pi_{4}^{T} \bar{Z}_{2} \Pi_{4} \\
+\operatorname{Sym}\left(\left(\tilde{e}_{1}^{T}+\varepsilon \tilde{e}_{4}^{T}\right) \breve{\Psi}^{T}\right)
\end{array}
$$

$$
\begin{gathered}
\bar{\Upsilon}_{2}=\operatorname{Sym}\left(\tilde{\Pi}_{1}^{T} \bar{X}_{1} \tilde{\Pi}_{3}\right)+\operatorname{Sym}\left(\tilde{e}_{1}^{T} \bar{X}_{2} \tilde{e}_{2}\right)+\tilde{\Pi}_{2}^{T} \hat{Q} \tilde{\Pi}_{2} \\
\bar{\Upsilon}_{3}=-\tilde{e}_{2}^{T} \hat{Q}_{1} \tilde{e}_{2}+\operatorname{Sym}\left(\Pi_{4}^{T} \bar{\Re}_{1} \Pi_{5}\right)+\Pi_{4}^{T} \bar{Z}_{1} \Pi_{4}
\end{gathered}
$$

$$
\begin{gathered}
\bar{\Upsilon}_{12}=\sqrt{h_{k}} \Pi_{4}^{T} \bar{N}_{1} \\
\bar{\Upsilon}_{13}=h_{U} \sqrt{h_{k}} \Pi_{4}^{T} \bar{N}_{2}
\end{gathered}
$$

with

$$
\begin{gathered}
\breve{\Psi}=\left[\begin{array}{llll}
A_{i} G^{T} & B_{i} T_{j} & 0 & -G^{T}
\end{array}\right] \\
\hat{Q}=\operatorname{diag}\{G, G\} \tilde{Q} \operatorname{diag}\{G, G\}^{T}
\end{gathered}
$$

where $\bar{Z}_{1}, \bar{Z}_{2}, \bar{X}_{1}, \bar{X}_{2}, \bar{N}_{1}, \bar{N}_{2}, R, \bar{\Re}_{1}$ and $\bar{\Re}_{2}$ have been defined in Theorem 2, and the gain matrix $K_{j}$ of the sampled-data controller without state quantization is defined as $K_{j}=T_{j} G^{-T}$.

Remark 5. The stability of the sampled-data T-S fuzzy system with and without the quantized state is investigated in Theorem 1 and Corollary 1 . And the upper bound of the sampling interval for T-S fuzzy system is larger than the existing results in Lam et al. (2007), Zhu et al. (2012), Wu et al. (2014b), and Liu et al. (2016), which will be proven in further simulation examples.

\section{NUMERICAL EXAMPLES}

This section provides two numerical examples to demonstrate the effectiveness and superiority of the proposed method.

Example 1. The Lorenz system with an input term (Wu et al., 2014 b) is given as follows

$$
\left\{\begin{array}{l}
\dot{x}_{1}(t)=-a x_{1}(t)+a x_{2}(t)+u_{1}(t) \\
\dot{x}_{2}(t)=c x_{1}(t)-x_{2}(t)-x_{1}(t) x_{3}(t) \\
\dot{x}_{3}(t)=x_{1}(t) x_{2}(t)-b x_{3}(t)
\end{array}\right.
$$

The Lorenz system (Equation 25) can be represented as a type of T-S fuzzy system (Equation 2) with the following parameters

TABLE 1 | Maximum allowable bounds $h$ without state quantization in Example 1.

Lam et al., 2007 Zhu et al., 2012 Wu et al., 2014b Liu et al., 2016 Corollary 2 $\begin{array}{llllll}h & 0.0158 & 0.0270 & 0.0347 & 0.0560 & 0.0741\end{array}$ 


$$
A_{1}=\left[\begin{array}{ccc}
-a & a & 0 \\
c & -1 & -d \\
0 & d & -b
\end{array}\right], A_{2}=\left[\begin{array}{ccc}
-a & a & 0 \\
c & -1 & d \\
0 & -d & -b
\end{array}\right], B_{1}=B_{2}=\left[\begin{array}{l}
1 \\
0 \\
0
\end{array}\right]
$$

and the membership functions satisfy $h_{1}\left(x_{1}(t)\right)=\left(1+\frac{x_{1}(t)}{2}\right) / 2$ and $h_{2}\left(x_{1}(t)\right)=1-h_{1}\left(x_{1}(t)\right)$.

Here we choose $a=10, b=8 / 3, c=28$, and $d=25$.

Case I: For the case with the quantized state, take the quantizer densities as

$$
\rho_{i}=1 / 2, i=1,2,3,
$$

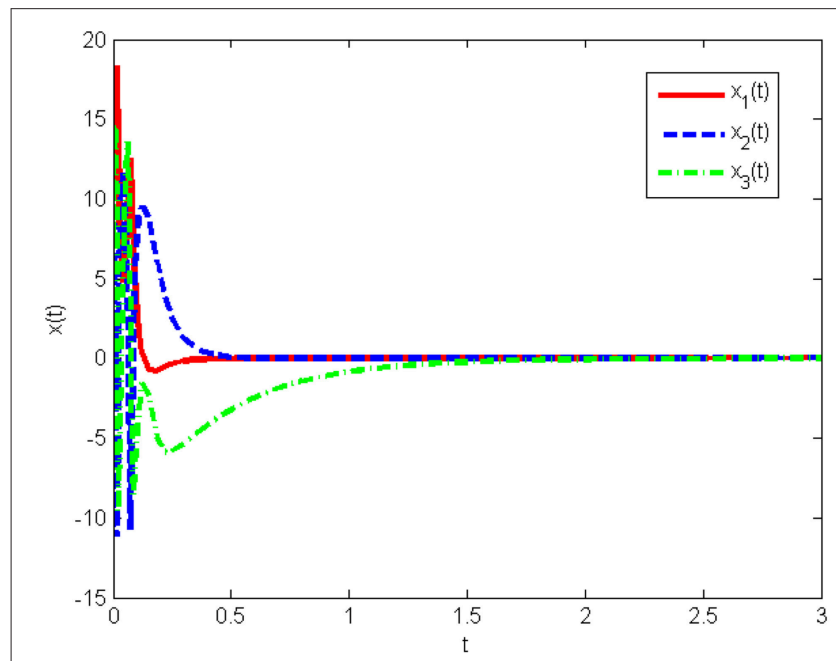

FIGURE 3 | State responses of system (Equation 25) without state quantization in Example 1.

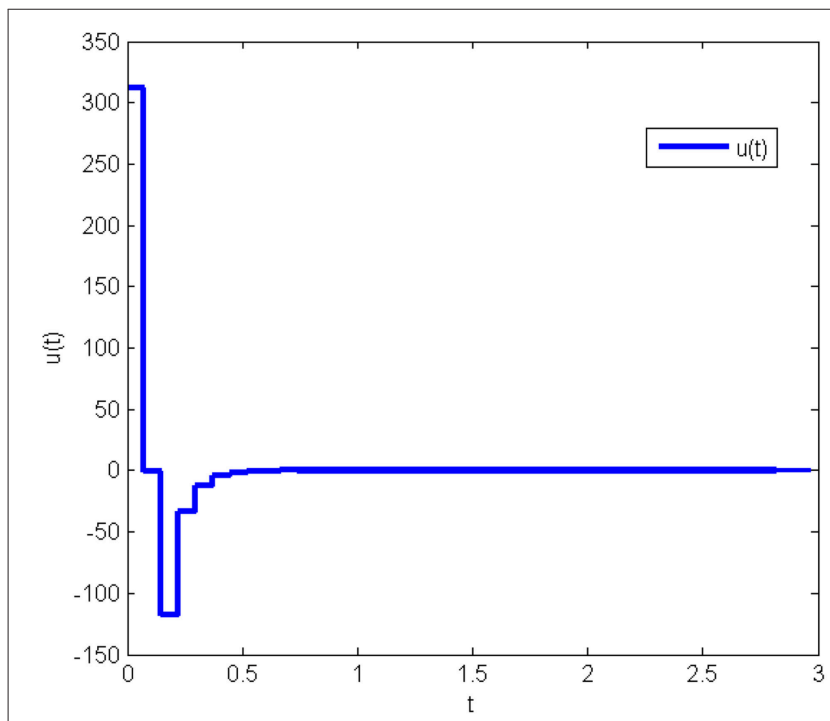

FIGURE 4 | Control input of system (Equation 25) without state quantization in Example 1. and the quantizer parameter is supposed as

$$
l_{m}=\left(1-\rho_{i}\right) /\left(1+\rho_{i}\right)=1 / 3
$$

Considering the quantized state based on Theorem 2, when $\varepsilon=$ 0.1 , the allowable maximum sampling period that can ensure the asymptotic stability of system (Equation 1) is 0.0742 , which is larger than 0.0503 obtained in Liu et al. (2016) actually, and the corresponding gain matrices are

$$
K_{1}=[-11.0256-15.249511 .5024]
$$

$$
K_{2}=[-11.0256-15.2495-11.5024]
$$

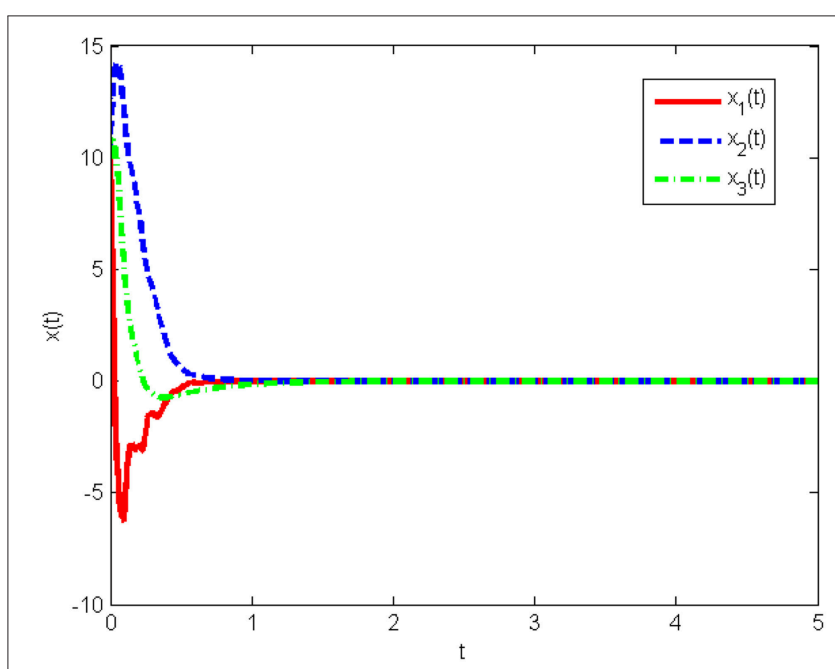

FIGURE $\mathbf{5}$ | State responses of system (Equation 26) with state quantization in Example 2.

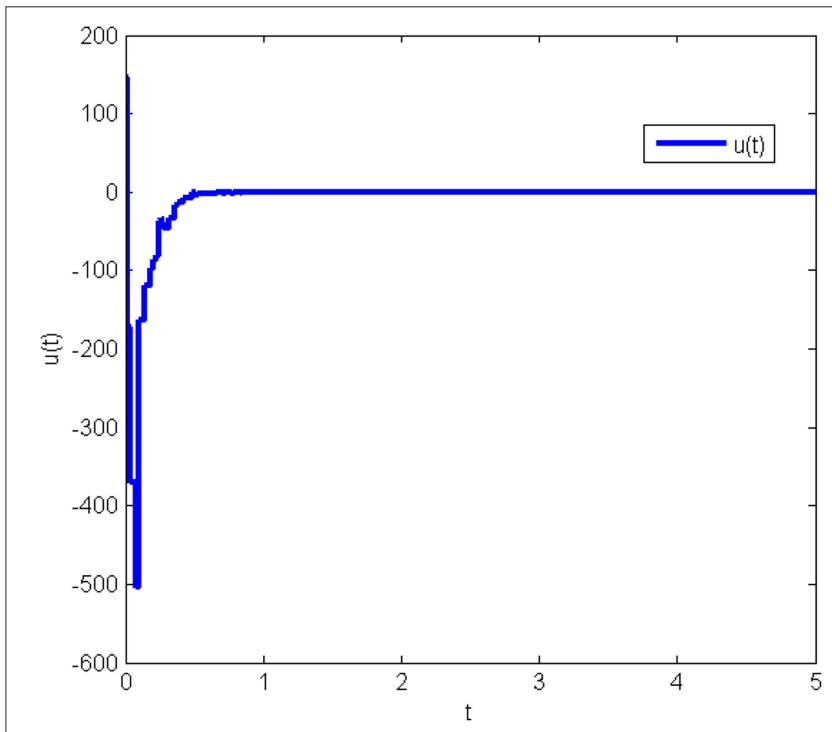

FIGURE 6 | Control input of system (Equation 26) with state quantization in Example 2. 
The response curves of system (Equation 25) under the initial condition $x(0)=\left[\begin{array}{lll}20 & 50 & 80\end{array}\right]^{T}$ with the obtained gain matrices are given in Figure 1, and the control input $u(t)$ is shown in Figure 2. This proves that the Lorenz system (Equation 25) with state quantization under the obtained sampled-data controller, in this work, is asymptotically stable.

TABLE 2 | Maximum allowable bounds $h$ without state quantization in Example 2.

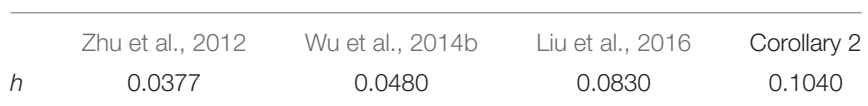

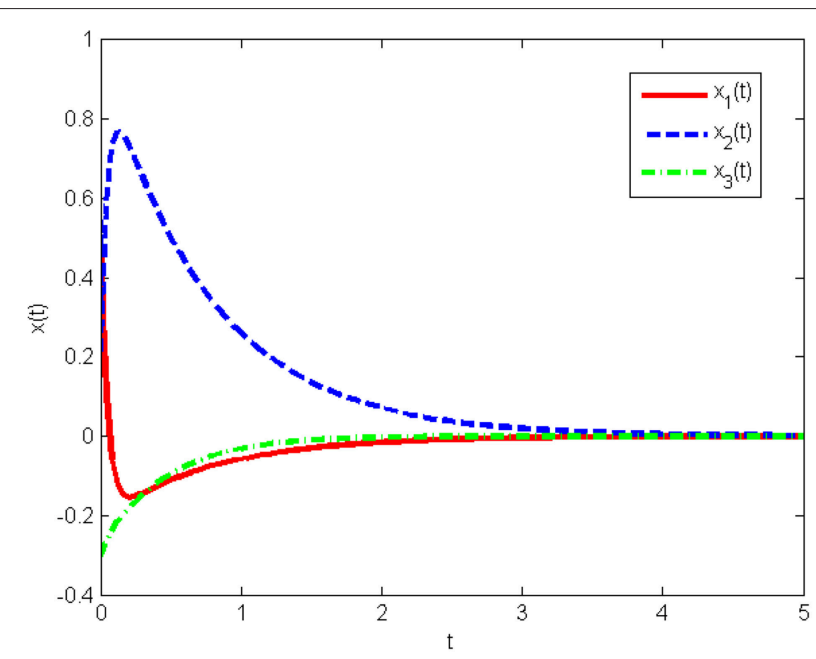

FIGURE 7 | State responses of system (Equation 26) without state quantization in Example 2.

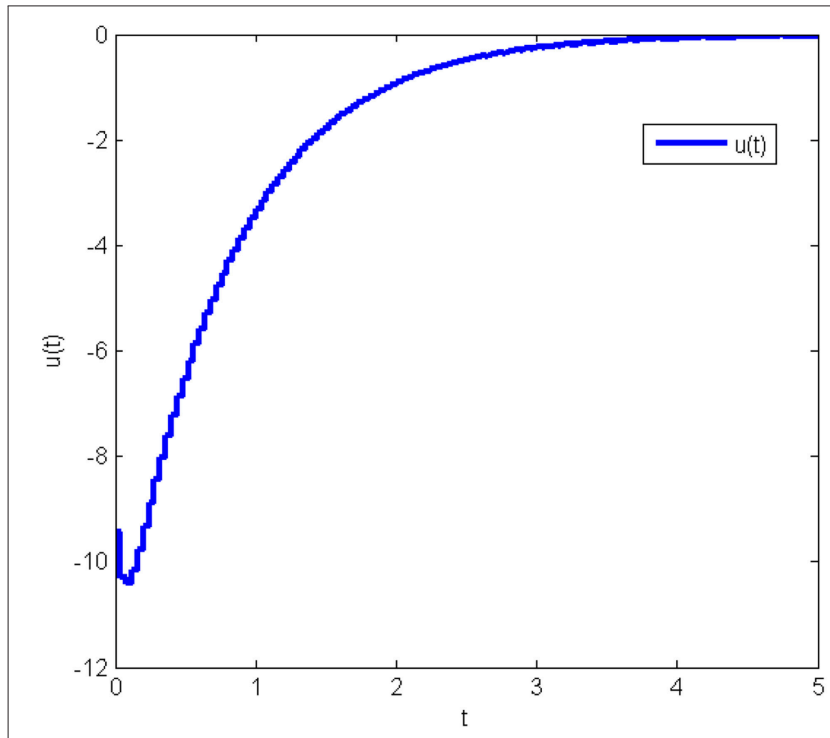

FIGURE 8 | Control input of system (Equation 26) without state quantization in Example 2.
Case II: For the case without state quantization, based on Corollary 2 , as $\varepsilon=0.1$, the allowable maximum sampling period ensuring the asymptotic stability of system (Equation 7) is 0.0741 . And the allowable upper bounds $h$ for the sampling interval are obtained, listed in Table 1. It can be seen that the upper bound for the sampling interval under the proposed method is larger than those obtained with existing methods in Lam et al. (2007), Zhu et al. (2012), Liu et al. (2016), and Wu et al. (2014b).

Simulation results are provided to verify the effectiveness of the proposed method. When $h=0.0741$, by solving LMIs (Equations 23, 24), we can obtain corresponding gain matrices as follows

$$
\begin{gathered}
K_{1}=[-11.2795-15.291011 .4783] \\
K_{2}=[-11.2795-15.2910-11.4783]
\end{gathered}
$$

The response curves of the system (Equation 25) with the initial condition $x(0)=\left[\begin{array}{lll}10 & 10 & 10\end{array}\right]^{T}$ under the obtained gain matrices are given in Figures 3, 4 shows the control input $u(t)$. This proves that the controller obtained in this work is correct and valid.

Example 2. The dynamic of unified chaotic system with an input term (Liu et al., 2016) is given as

$$
\left\{\begin{array}{l}
\dot{x}_{1}(t)=-(25 a+10)\left(x_{1}(t)-x_{2}(t)\right)+u_{1}(t) \\
\dot{x}_{2}(t)=(28-3 a) x_{1}(t)+(29 a-1) x_{2}(t)-x_{1}(t) x_{3}(t) \\
\dot{x}_{3}(t)=x_{1}(t) x_{2}(t)-(8+a) x_{3}(t) / 3
\end{array}\right.
$$

Note that the unified chaotic system (Equation 26) with $x_{1}(t) \in$ $[-d, d]$ can be represented in the T-S fuzzy system (Equation 2) with

$$
\begin{gathered}
A_{1}=\left[\begin{array}{ccc}
-(25 a+10) & 25 a+10 & 0 \\
28-3 a & 29 a-1 & -d \\
0 & d & -(8+a) / 3
\end{array}\right] \\
A_{2}=\left[\begin{array}{ccc}
-(25 a+10) & 25 a+10 & 0 \\
28-3 a & 29 a-1 & d \\
0 & -d & -(8+a) / 3
\end{array}\right] \\
B_{1}=B_{2}=\left[\begin{array}{l}
1 \\
0 \\
0
\end{array}\right]
\end{gathered}
$$

and the membership functions are $h_{1}\left(x_{1}(t)\right)=\left(d+x_{1}(t)\right) / 2 d$ and $h_{2}\left(x_{1}(t)\right)=\left(d-x_{1}(t)\right) / 2 d$, respectively.

Here $a=0.2$ and $d=10$ are chosen, and two cases with and without state quantization are considered further.

Case I: Taking the same quantizer densities as in Example 1, and using Theorem 2, when $\varepsilon=0.01$, system (Equation 26) can be asymptotically stable with the maximum sampling period $h=0.0535$, which is larger than 0.0424 in Liu et al. (2016). Then, we choose $h=0.02$, and by solving LMIs (Equations 18, 19), the corresponding gain matrices can be obtained as follows

$$
K_{1}=[-25.4237-30.89792 .6211]
$$




$$
K_{2}=[-25.4237-30.8979-2.6211]
$$

Under the obtained gain matrices and the initial condition $x(0)=$ [10 1010$]^{T}$, the state response and control input $u(t)$ for system (Equation 26) are shown in Figures 5, 6, respectively. This proves that the controller obtained in this work is correct and valid.

Case II: The maximum allowable upper bound of the sampling interval $h$ without state quantization under the sampled-data control is listed in Table 2, which shows that the results in Corollary 2 is superior to existing ones.

When $\varepsilon=0.1$ and $h=0.04$, by solving LMIs (Equations 23, $24)$, we can obtain the corresponding gain matrices as follows

$$
\begin{gathered}
K_{1}=[-11.7978-20.67591 .2851] \\
K_{2}=[-11.7978-20.6759-1.2851]
\end{gathered}
$$

The response curves of system (Equation 26) with initial condition $x(0)=\left[\begin{array}{lll}0.5 & 0.2-0.3\end{array}\right]^{T}$ under the above gain matrices are displayed in Figure 7, and the corresponding control input $u(t)$ is given in Figure 8. This proves that the controller proposed in Corollary 2 can ensure the asymptotic stability of the T-S fuzzy sampled-data system (Equation 26) without state quantization.

\section{CONCLUSIONS}

In this work, we have investigated the stability for a class of nonlinear T-S fuzzy sampled-data systems with state quantification. A new LKF approach has been constructed

\section{REFERENCES}

Chen, T. W. and Francis, B. (2012). Optimal Sampled-Data Control Systems. Berlin: Springer Science, Business Media.

Dong, S., Su, H. and Shi, P. (2017a). Filtering for discrete-time switched fuzzy systems with quantization. IEEE Trans. Fuzzy Syst. 25, 1616-1628. doi: 10.1109/TFUZZ.2016.2612699

Dong, S., Wu, Z.G., Shi, P., Su, H. and Lu, R. (2017b). Reliable control of fuzzy systems with quantization and switched actuator failures. IEEE Trans. Syst. Man Cybern. Syst. 47, 2198-2208. doi: 10.1109/TSMC.2016. 2636222

Fridman, E. (2010). A refined input delay approach to sampled-data control. Automatica 46, 421-427. doi: 10.1016/j.automatica.2009.11.017

Fridman, E., Seuret, A., and Richard, J. P. (2004). Robust sampled-data stabilization of linear systems: an input delay approach. Automatica 40, 14411446. doi: 10.1016/j.automatica.2004.03.003

$\mathrm{Fu}, \mathrm{M}$. and Xie, L. (2005). The sector bound approach to quantized feedback control. IEEE Trans. Automatic Control 50, 1698-1711. doi: 10.1109/TAC.2005.858689

Hu, L. S., Bai, T., Shi, P., and Wu, Z. M. (2007) Sampled-data control of networked linear control systems. Automatica 43, 903-911. doi: 10.1016/j.automatica.2006.11.015

Lam, H. K., and Leung, F. H. F. (2007). Stabilization of chaotic systems using linear sampled-data controller. Int. J. Bifurcat. Chaos 17, 2021-2031. doi: 10.1142/S0218127407018191

Lee Tae H., and Park Ju H. (2017). Stability analysis of sampled-Data systems via free-matrix-based time-dependent discontinuous lyapunov approach. IEEE Trans. Automatic Control 62, 3653-3657. doi: 10.1109/TAC.2017. 2670786 and a Free-Matrix-Based boundary treatment technique for integral inequalities has been adopted in order to obtain less conservative stability conditions and correspondingly, a controller has been designed. Furthermore, the stability of the T-S fuzzy sampled-data system without quantized states, has also been discussed and sampled-data controllers have been designed accordingly. The experimental results show that the maximum sampling interval for T-S fuzzy sampled-data systems with and without quantized states in our work, are both larger than the results in previous studies. Nevertheless, some other interesting problems that need to be addressed still exist, such as the reliable control design for the sampleddata T-S fuzzy systems with state quantization, and the extension of our developed approaches to the dissipativitybased sampled-data control design, which deserve further investigation.

\section{AUTHOR CONTRIBUTIONS}

All authors listed have made a substantial, direct and intellectual contribution to the work, and approved it for publication.

\section{ACKNOWLEDGMENTS}

This work was supported by the Natural Science Foundation of China under Grant 61503045, 61603121, National Natural Science Foundation of Jilin Province under Grant 20180101333JC.
Li H., Gao Y.,Wu L., and Lam H.K. (2015). Fault detection for T-S fuzzy time-delay systems: Delta operator and input-output methods. IEEE Trans. Cybernetic. 45, 229-241. doi: 10.1109/TCYB.2014.2323994

Liu K., Seuret A., and Xia Y. (2017). Stability analysis of systems with timevarying delays via the second-order Bessel-Legendre inequality. Automatica 76, 138-142. doi: 10.1016/j.automatica.2016.11.001

Liu, Y., and Lee, S. M. (2016). Stability and stabilization of Takagi-Sugeno fuzzy systems via sampled-data and state quantized controller. IEEE Trans. Fuzzy Syst. 24, 635-644. doi: 10.1109/TFUZZ.2015.2469099

Niu, Y., Jia, T., Wang, X. and Yang, F. (2009). Output feedback control design for NCSs subject to quantization and dropout. Inf. Sci. 179, 3804-3813. doi: 10.1016/j.ins.2009.07.006

Shao, H., Han Q. L., Zhang, Z. and Zhu X. (2014). Sampling-interval-dependent stability for sampled-data systems with state quantization. Int. J. Robust Nonlinear Control 24, 2995-3008. doi: 10.1002/rnc.3038

Tanaka, K., and Wang, H. O. (2004). Fuzzy Control Systems Design and Analysis: A Linear Matrix Inequality Approach. New York, NY: John Wiley Sons.

Wang S., Feng J., and Jiang Y. (2016). Input-output method to fault detection for discrete-time fuzzy networked systems with time-varying delay and multiple packet losses. Int. J. Syst. Sci. 47, 1495-1513. doi: 10.1080/00207721.2014.938785

Wang S., Ji W., Pang J., and Jiang Y. (2018a). Dissipativity-based sampled-data reliable control design for T-S fuzzy system using limited bessel-legendre inequality. IEEE Access 6, 73405-73415. doi: 10.1109/access.2018.2882125

Wang S., Jiang Y., and Li Y. (2018b). Distributed $H_{\infty}$ consensus fault detection for uncertain T-S fuzzy system with time-varying delays over lossy sensor networks. Asian J. Control 20, 2171-2184. doi: 10.1002/asjc.1721

Wang S., Jiang Y., Li Y., and Liu D. (2017). Fault detection and control codesign for discrete-time delayed fuzzy networked control systems subject 
to quantization and multiple packet dropouts. Fuzzy Sets Syst. 306, 1-25. doi: 10.1080/00207721.2012.658882

Wu Z., Shi P., and Su H. (2014a). Sampled-data fuzzy control of chaotic systems based on a T-S fuzzy model. IEEE Trans. Fuzzy Syst. 22, 153-163. doi: $10.1177 / 0142331217740420$

Wu, Z., Shi P., Su H., and Lu R. (2015). Dissipativity-based sampleddata fuzzy control design and its application to truck-trailer system. IEEE Trans. Fuzzy Syst. 23, 1669-1679. doi: 10.1109/TFUZZ.2014.23 74192

Wu, Z. G., Shi, P., Su, H., and Chu, J. (2014b). Sampled-data fuzzy control of chaotic systems based on a T-S fuzzy system model. IEEE Trans. Fuzzy Syst. 22, 153-162. doi: 10.1109/TFUZZ.2013.2249520

Xia, Y., Yang, H., Shi, P., and Fu, M. (2010). Constrained infinite-horizon model predictive control for fuzzy-discrete-time systems. IEEE Trans. Fuzzy Syst. 18, 429-436. doi: 10.1109/TFUZZ.2010.2043441

Yang, F., Zhang, H. and Wang, Y. (2014). An enhanced input-delay approach to sampled-data stabilization of T-S fuzzy systems via mixed convex combination. Nonlinear Dynam. 75, 501-512. doi: 10.1007/s11071-0131080-8

Zhao, J., Lin, C.-M., and Chao, F. (2019). Wavelet fuzzy brain emotional learning control system design for MIMO uncertain nonlinear systems. Front. Neurosci. 12:918. doi: 10.3389/fnins.2018.00918

Zhao, Y., Gao, H., Lam, J., and Du, B. (2009). Stability and stabilization of delayed T-S fuzzy systems: a delay partitioning approach. IEEE Trans. Fuzzy Syst. 17, 750-762. doi: 10.1109/TFUZZ.2008. 928598
Zhong, Z., Zhu, Y., and Lam, H. K. (2018). Asynchronous piecewise output-feedback control for large-scale fuzzy systems via distributed event-triggering schemes. IEEE Trans. Fuzzy Syst. 26, 1688-1703. doi: 10.1109/TFUZZ.2017.2744599

Zhu Y. Z., Zhong, Z. X., Basin, Michael V., and Zhou, D. H. (2018a). A descriptor system approach to stability and stabilization of discrete-time switched PWA systems. IEEE Trans. Autom. Control 2018, 3456-3463. doi: 10.1109/TAC.2018.2797173

Zhu Y. Z., Zhong, Z. X., Zheng, W. X., and Zhou, D. H. (2018b). HMM-based $H_{\infty}$ filtering for discrete-time markov jump LPV systems over unreliable communication Channels. IEEE Trans. Syst. Man Cybernetic. Syst. 48, 20352046. doi: 10.1109/TSMC.2017.2723038

Zhu, X., Chen, B., Yue, D. and Wang, Y. (2012). An improved input delay approach to stabilization of fuzzy systems under variable sampling. IEEE Trans. Fuzzy Syst. 20, 330-341. doi: 10.1109/TFUZZ.2011.2174242

Conflict of Interest Statement: The authors declare that the research was conducted in the absence of any commercial or financial relationships that could be construed as a potential conflict of interest.

Copyright (c) 2019 Wang, Chen, Ji and Liu. This is an open-access article distributed under the terms of the Creative Commons Attribution License (CC BY). The use, distribution or reproduction in other forums is permitted, provided the original author(s) and the copyright owner(s) are credited and that the original publication in this journal is cited, in accordance with accepted academic practice. No use, distribution or reproduction is permitted which does not comply with these terms. 